\title{
REMARKS ON THE COGNITIVE BASE OF PRAGMATIC PRINCIPLES*
}

\author{
ANDRÁS KERTÉSZ - CSILLA RÁKOSI
}

\begin{abstract}
The present paper aims at the exemplification of the applicability of plausibility analysis to linguistics. Starting from the criticism of Robinson (1997), the paper argues for two assumptions. Firstly, as opposed to a theory of distributed systems, it is a theory of plausible reasoning that is capable of capturing basic methodological problems of theory formation in pragmatics (such as circularity, category error, the arbitrariness of interpretations of data and the objectification of the theorist's cultural and linguistic knowledge as principles of language behaviour). Secondly, the cognitive base of pragmatic principles is inferential and plausibilistic, rather than non-inferential and probabilistic.
\end{abstract}

\section{Introduction}

\subsection{On objective theories of language and cognition}

In a provocative paper Edward A. Robinson (1997) claims that pragmatic theories motivated by Grice (1975) or Sperber and Wilson's relevance theory (1986/1995) are paradigm examples of what he calls "objective theories of language and cognition". This kind of objectivism has, in Robinson's view, at least two central features (see Robinson 1997, 256):

(a) Researchers presuppose the distinction between subject and object which is characteristic of the relationship between a scientist and the things he or she observes. This means that scientists assume to observe impartially external objects. As a result, it is taken for granted that the rules and structures described are impartial facts which are true or false.

(b) Objective theories of language and cognition tend to turn the structures which have been created in this way into structures of the

* Work on the present paper was supported by the Research Group for Theoretical Linguistics of the Hungarian Academy of Sciences at the University of Debrecen. We are grateful to Károly Bibok, János László, Enikő Németh T. and two anonymous reviewers for helpful comments. Our special thanks are due to George Seel for improving our English. None of these people are responsible for the shortcomings of our paper.

1216-8076/\$20.00 (C) 2005 Akadémiai Kiadó, Budapest 
mind. They assume the existence of a kind of cognitive content which causes people to act and which can be described independently of the background knowledge of the scientists who observe linguistic behaviour.

Objective theories in this sense raise a series of fundamental difficulties that can be summarized as follows (see Robinson 1997, 256-8).

The first is that objective theories yield a category error according to which the mechanisms underlying the linguistic behaviour described are identified with the models of language one obtains as a result of trying to objectively describe the things observed. That is, the theoretical model is assumed to be identical with the cognitive content underlying linguistic behaviour which this model is intended to describe. In this way objective theories are assumed to translate immediately into mental structures.

Secondly, from (a) and (b) above it follows that objective theories of cognition are in fact nothing but the interpretations of the scientist.

Thirdly, a certain kind of circularity presents itself. On the one hand, the theorist maintains that there exist certain principles which govern communicative behaviour. On the other hand, theorists use their cultural and linguistic knowledge in order to define basic terms with the help of which communicative behaviour is described. Accordingly, what the theorist describes is in fact the reflection of his/her own communicative and cultural knowledge. ${ }^{1}$ By way of illustration, let us mention the example of the Gricean maxims. At first sight it seems to be the case that these maxims objectively describe certain mechanisms which seem to underlie everyday communication and which may also account for certain kinds of meanings that are brought about by certain utterances. Nevertheless, these maxims can be formulated only because the researcher himself/herself has an understanding of what in a given society counts as brief, relevant, orderly, informative. Thus, what happens is that the researcher's own cultural knowledge is objectified: those pieces of information which are properties of the theory are assumed to constitute objective accounts of what actually happens in communicative processes. Such knowledge is considered to be the cognitive base of pragmatic principles, while in fact it is nothing but cultural knowledge that all members of a given society, including the researcher himself/herself, share. The researcher does not detect regularities objectively given, but rather, he/she projects his/her own knowledge onto the object of his/her

\footnotetext{
${ }^{1}$ Independently of Robinson's argumentation, Kertész (2004a) discusses a similar kind of circularity with respect to cognitive semantic theories such as Lakoff and Johnson's embodied realism and Bierwisch and Lang's two-level model.
} 
investigations while claiming the latter to be objectively detected. ${ }^{2}$ This inevitably leads to a difficulty which concerns the infinity of the relations included in the base:

"Of course, it can be argued that this simply means we need to objectively define all of the relationships within the cultural knowledge base and to allow the principles to act on these defined relationships. [...] In an objective theory, all of the conceptual base that can make a difference in interpretation must be explicitly defined. But, if we consider this, we find that the number of relations that need to be explicated is theoretically infinite. For instance, consider (3):

(3) A: How are we getting to the movie?

B: Well, I have the car.

For B's response to mean 'I'll drive', B must have a licence, the car must have fuel, it must seat more than one passenger, the battery must have acid, all the essential working parts must be present and correctly assembled, the laws of physics must be in effect, etc., ad infinitum. Any of these facts of cultural knowledge can be challenged to cancel the implicature in B's response that they can use the car. [...] Because all of these assumptions can figure in the final implicature, an objective system must specify all of these assumptions in all of the different situations in order to determine which are in effect for a given utterance. Once we have taken for granted the epistemological assumption inherent in objective approaches to language and cognition, we must fully formalize the knowledge base. Of course, to define all these possible inferences, there must be a fully spelled-out knowledge base.

The complexity of this knowledge base has been a central concern for many theorists in the behavioural and cognitive sciences. [...] Ultimately, the task proved to be impossible - objectively defining the full cultural base of understanding is an infinite process of specifying and respecifying finer and finer grains of detail. [...] It is beyond the ability of a formal system to capture cultural knowledge, i.e., the knowledge used in discourse understanding." (Robinson 1997, 258-9; emphasis added)

The difficulties thus summarized boil down to two distinct but interrelated problems. The first is a metascientific problem concerning the methodology which pragmatic theories make use of, whereas the second is an object-scientific one which focuses on a specific aspect of the object of pragmatic theories, namely, the cognitive base of pragmatic principles:

${ }^{2}$ Cf. the following quotation: "As a cognitive mechanism, these principles are defined as decision metrics. What a brief or orderly usage is, is what the principle is supposed to tell us. But, to make a decision, these principles must have a definition of the value they are determining. Unfortunately, this definition is a reflection of the structure of the theorist's knowledge." (Robinson 1997, 258; emphasis added) 
(P1) The methodological problem of pragmatic theories: How can a pragmatic theory avoid

(a) the category error previously referred to,

(b) the potential arbitrariness of interpretations,

(c) circularity, and

(d) that the theorist's cultural and linguistic knowledge be objectified as principles of language behaviour?

(P2) The problem of the cognitive base of pragmatic theories: How can the cognitive base underlying pragmatic principles be accounted for, if we know that it

(a) contains an infinite amount of assumptions,

(b) consists of assumptions which are context-dependent, and

(c) cannot be captured by objective theories because the latter should account for an infinite process of specifying and respecifying the information it consists of?

\subsection{Solutions to (P1) and (P2)}

Robinson's solution to (P1) is this:

(S1) A theory of distributed systems avoids (P1)(a)-(d).

In accordance with (S1), he also puts forward a possible solution to (P2):

"The regularities which the theories above describe emerge from the action of a distributed system as it exists in the world. They are not represented in the cognitive system but reflect its general existential nature. They represent an external observer's view of the overall activity of a number of independent but mutually sensitive interacting cognitive and situational factors, none of which independently represents any specific pragmatic principle. [...] Rather than viewing concepts as discrete representations, we should view them as relative and subjective entities. In such a view, internal representations are dynamic, generalized associations which always act relative to the environment. [...] I will relate objective linguistic theories to a more realistic view of cognition which does not depend on individual mental representations and calculations. [...] This approach, while it remains objective as an external description of behaviour, has the advantage of not utilizing locally definable representations. Instead, it treats the speaker as an integral part of the environment by modelling the environment and the associational patterns and mechanisms that tie the individual to the environment. We must not treat mental representations as descriptions but as probabilistic traces tied to the environment. Understanding is not the calculation and representation 
of a knowledge structure in the mind. It is rather the state of the cognitive system at a certain point in time in relation to the world around it." (Robinson 1997, 259f; emphasis added)

The main aspects of this proposal can be summarized as in (S2):

(S2) The sub-problems of (P2) can be solved by assuming that the cognitive base of pragmatic principles includes relations which are

(a) non-inferential, and

(b) probabilistic.

\subsection{Remarks on (S1) and (S2)}

There are a couple of important issues which seem to question the workability of Robinson's model. Let us in what follows point out some of them.

The model proposed by Robinson cannot avoid the problems listed in (P1) as he himself observes. ${ }^{3}$ Nevertheless he maintains that "whereas the pragmatic theories [...] point towards regularities at their level of description, this model can be used to point to regularities at another, perhaps neural or ecological, level of description" (Robinson 1997, 264).

Thus, Robinson simply pushes the problem to another level of theoretical description, i.e., to another subsystem of "a distributed system" (e.g., perceptual system, associative memory). However, we cannot carry on the generation of new levels endlessly; in order to prevent ourselves from getting into an infinite regress we have to stop somewhere. But at the last level we are forced to turn the structures of the theoretical description into structures of the mind.

Moreover, if we ask the question of how he knows which conceptual content is associated with a certain utterance, we cannot avoid answering: on the basis of his cultural and linguistic knowledge. So, the same kind of circularity seems to appear in this model as that which it was intended to resolve. This can be illustrated by the following quotation: ${ }^{4}$

3 "Of course, we can formulate objections against this model similar to those made earlier against the linguistic descriptive models." (Robinson 1997, 264)

${ }^{4}$ The quotation refers to the following example:

"(2) A: Did you go to Bloomingdale's when you went to New York?

B: Well, what do you think of my new dress?" (Robinson 1997, 257)

Acta Linguistica Hungarica 52, 2005 
"For example, in (2) the conversants are discussing whether B had gone to Bloomingdale's. Thus, knowledge associated with Bloomingdale's is active for both conversants. Part of this knowledge is the fact that Bloomingdale's sells dresses. This is simply part of the cultural knowledge that A and B possess about Bloomingdale's. These two individuals, having been raised in their particular culture, have learned what Bloomingdale's is, and part of this knowledge is that it sells dresses. While this association may not be the most active thing for $\mathrm{A}$ at the time of his or her utterance, this relationship is further activated by B's response. Furthermore, A's goal is to get an answer to the first question, and $\mathrm{A}$ and $\mathrm{B}$ are following standard conversational patterns. So the implicature is automatically available to A because of the correspondence between A's goals and salient aspects of the conceptual material associated with dresses and Bloomingdale's. Within this approach, it is not calculated; it arises from the correspondence of all of these parts as they are activated within this context." (op.cit., 267f; emphasis added)

Now we may ask what the source of the knowledge of the associations is that are most probable in this situation: surely, it is Robinson's own cultural knowledge. He does not mention any experiments in which the perceptual system and the memory of the people in this dialogue have been investigated with the methods of neuropsychology. Therefore, (S1) is not capable of capturing this kind of problem (see also section 1.1).

At least one of the sources of the difficulties might be that the gap between the level of the associative memory and that of linguistic behaviour is too great. For instance, the principle that "[...] if we look at how the cognitive system [...] works, we see that relevance is actually created by this system, because it is organized to associate things related in previous experience" (ibid., 267), significantly underdetermines linguistic meaning.

In direct connection with this finding, (S2)(a) appears to be problematic as well. Suppose that there is a third person $\mathrm{C}$ who has never heard the name Bloomingdale's. Despite this fact, he or she may find out the implicated content of B's answer - without having any knowledge associated with the name of the department store mentioned. In this case, we cannot claim that the implicature is automatically available to him or her. On the contrary: C can only 'calculate' the intended meaning of B's utterance by drawing inferences from the content of the question, the answer and some additional background knowledge about buying dresses. A similar case can be constructed through the modification of the dialogue:

(1) A: Your dress is really nice.

B: I was at Bloomingdale's when I went to New York.

Acta Linguistica Hungarica 52, 2005 
Here A need not have any knowledge of Bloomingdale's to be able to understand the whole content of B's response. He or she can only infer it from pieces of information rooted in his or her cultural knowledge. Consequently, (S2)(a) may be questioned.

A third example is worth mentioning:

(2) A: Mrs. X is an old bag.

B: The weather has been quite delightful this summer, hasn't it? ${ }^{5}$

If we consider the principle formulated by Robinson that " [...] only what is specifically uttered is what is fully activated" (ibid., 265f), then we cannot find out what B intended to communicate. Certainly, A's statement is part of the context, so we have to take it into consideration as well. But B's utterance has seemingly nothing to do with A's words: it seems to be totally irrelevant in comparison to the content of A's statement. Realizing this, we remember previous experiences of similar situations and conclude that $\mathrm{B}$ wanted to say that $\mathrm{A}$ had been impolite and he or she refused to talk in this way. But this means that A can be in possession of the entire situation only after discovering that B's contribution has been irrelevant. Consequently, the link between associations and implicatures seems to be not as directly probabilistic as supposed by Robinson - therefore, (S2)(b) is problematic, too. Rather, it seems to be the case that we are dealing with a 'cyclic' and 'prismatic' revaluation of the pieces of information available.

The point, then, is that the claim that "the activation of the goal and of the perceived circumstances at the same time results in a correspondence between all of the parts which arises as an automatic, reflexive mental process [...]" (Robinson 1997, 268) might be replaced by the following assumption: The activation of the goal and of the perceived circumstances at the same time results in a correspondence between all of the parts which arises as a cyclic and prismatic inference and decision process.

In sum, the above considerations indicate that both (S1) and (S2) may be problematic and these problems justify the search for alternative suggestions.

\footnotetext{
${ }^{5}$ See Grice $(1975,54)$.
} 


\subsection{Alternatives to (S1) and (S2)}

At this point of our argumentation we hypothetically put forward the following alternatives to (S1) and (S2):

$\left(\mathrm{S}^{\prime}\right)$ A theory of plausible reasoning avoids (P1)(a)-(d).

$\left(\mathrm{S} 2^{\prime}\right)(\mathrm{P} 2)$ can be solved by assuming that the cognitive base of pragmatic principles includes relations which are

(a) inferential in a specific sense, and

(b) cyclic and prismatic.

As the above summary witnesses, at first sight Robinson's views seem to be quite odd, and therefore, one may ask the question if the problems (P1) and (P2) are 'real' problems, or rather, just arbitrarily constructed quandaries. Nevertheless, we will try to show that this is not so and that it is precisely the unusual nature of Robinson's line of argumentation that makes it an instructive starting point for illustrating the workability of our approach to the methodology of pragmatics both on an object- and metascientific level. We hope that in arguing for an alternative solution to $(\mathrm{P} 1)$ and $(\mathrm{P} 2)$ we will be able to sketch the basic tenets of a theory of plausible reasoning which will prove to be a fruitful tool for solving central problems of theory formation in pragmatics in particular and linguistics in general.

In the rest of this paper we will proceed as follows. In section 2 we will briefly outline some tenets of a theory of plausible reasoning on a high level of abstraction (section 2.1); nevertheless, we will also show that it is not unreasonable to assume the cognitive reality of plausible inferences (section 2.2). In section 3 we will try to show that $\left(\mathrm{S} 2^{\prime}\right)$ is a possible alternative to (S2) which is capable of capturing (P2) by accounting for some of the shortcomings of (S2). In section 4 we will briefly touch on (P1) indicating that the theory of plausible reasoning we have introduced can maintain $\left(\mathrm{S}^{\prime}\right)$. Finally, section $\mathbf{5}$ will summarize the main findings and raise open questions that seem to be worth considering in the future. 


\section{On plausible inferences}

\subsection{Basic assumptions}

In this section we will summarize certain aspects of a possible theory of plausible reasoning. The scope of the present paper does not allow us to provide a precise explication of the basic notions, therefore we will consciously use them preexplicatively. Nevertheless, we make the following terminological remarks, which are not intended to be precise explications but which serve to render our line of argumentation followable and understandable.

First, the notions of 'deductive', 'conclusive', 'demonstrative' and 'logical' inference are used as synonyms, and so are the notions of 'plausible', 'non-demonstrative' and 'non-conclusive' inference. ${ }^{6}$ Second, we regard the basic concepts and the notational conventions of propositional logic as given, and they are not introduced systematically. Third, the notions of 'consistency' and 'non-contradiction' and those of 'inconsistency' and 'contradiction' are also treated as synonyms, respectively. Fourth, by 'heuristics' we understand rules (i) the role of which is to survey (in a non-systematic manner) relatively large domains of problems, (ii) which may lead to the solution of a certain problem, but (iii) which do not necessarily lead to the solution or to the optimal solution of this problem. Fifth, by 'data' we understand such assertions that embody the knowledge available to us for the solution of a problem in a given informational state (according to this formulation, we call not only those assertions 'data' which describe 'facts' or 'observations' in whatever sense of these terms, but also every such background assumption which we use during the argumentation). ${ }^{7}$

\footnotetext{
${ }^{6}$ Nevertheless, see Kertész - Rákosi (2005b) for a more sophisticated treatment of these notions according to which non-demonstrative/non-conclusive inferences include both plausible and fallacious inferences.

${ }^{7}$ On this interpretation of 'data' cf. Rescher and Brandom's characterization: "'Data': theses that can serve as acceptance-candidates in the context of inquiry, contentions which, at best, are merely presumptively true [...]. These are not certified truths (or even probable truths) but theses that are in a position to make some claims upon us for acceptance: They are prima facie truths in the sense that we would incline to grant them acceptance-as-true if (and this is a very big IF) there were no countervailing considerations upon the scene." (RescherBrandom 1979, 69)
} 
Presupposing these terminological remarks, in what follows we characterize plausible inferences by taking the classical work of George Polya and Nicholas Rescher as our starting point. ${ }^{8}$ We will integrate findings which have already been put forward in the literature and as a result of this integration of classical approaches we will sketch a coherent and many-sided account of plausible reasoning. ${ }^{9}$

(i) The notion of plausible inference. The common features of plausible inferences can be summed up in two points as a first approximation:

"First, they do not have the certainty of a strict demonstration. Second, they are useful in acquiring essentially new knowledge, and even indispensable to any not purely mathematical or logical knowledge, to any knowledge concerned with the physical world. We could call the reasoning that underlies this kind of evidence 'heuristic reasoning' or 'inductive reasoning' or (if we wish to avoid stretching the meaning of existing terms) 'plausible reasoning'." (Polya 1948, 221f; emphasis added)

The fundamental difference between plausible and deductive inferences can be demonstrated through the following example:

Deductive inference:

If $A$, then $B$

not $B$

not $A$

modus tollens

\section{Plausible inference:}

It is certain that if $A$ then $B$

$A$ is possible, $B$ is not certain

After verifying $B, A$ is more plausible

reduction

${ }^{8}$ It is important to emphasize that Polya's and Rescher's views are fully compatible: "Polya's entire analysis of the logic of inductive reasoning can also be accommodated on the present approach" (Rescher 1976, 67). Moreover, there are at least two substantial reasons for concentrating on these classical approaches. Firstly, due to the pioneering nature of these works, there is no avoiding their use in presenting the essentials of plausible reasoning (see Woods et al. 2000, 258). Secondly, Polya's and Rescher's ideas are not outmoded at all, because they have been rediscovered and integrated into current trends which revaluate traditional problems of the philosophy of science such as the distinction between the context of justification and the context of discovery, inconsistency, the process of scientific problem solving, etc.; in this way these ideas have been integrated into current approaches to argumentation theory and AI research. Therefore, it is unavoidable to take those classical works by Polya and Rescher as our starting point which most current accounts of plausible reasoning are rooted in. Should our approach turn out to be tenable, later refinements in the light of recent advances will be both possible and necessary.

${ }^{9}$ For a considerably more comprehensive discussion of our approach see Kertész (2004b); Kertész - Rákosi (2005a) and Rákosi (2005).

Acta Linguistica Hungarica 52, 2005 
(ii) The uncertainty of plausible inferences. The definitive difference between the two conclusions in the example above is that while with deductive inferences the truth of the conclusion follows from the truth of the premises with certainty, in the case of plausible inferences the premises merely increase the credibility of the conclusion (cf. Polya 1954, 113). Consequently, plausible inferences are less reliable by nature than conclusive inferences: they necessarily involve the possibility of mistakes, errors and rejectability (see also Walton 2001, 159).

(iii) The heuristic function of plausible inferences. We very often find ourselves in a situation during the solving of a problem that, at a certain point, we have several hypotheses (conjectures) at our disposal which mutually exclude each other, but every one of which is supported by certain considerations and therefore each may represent a possible alternative in view of the amount of information which we possess. Then we have to decide between competing hypotheses but we cannot turn to deductive logic for help. Thus, plausible inferences are heuristic tools with the purpose of bringing us closer to the solution of a certain problem, inasmuch as they help us form an opinion of which possible alternative is the most promising on the basis of the information available to us at any given moment (Polya 1948, 102; cf. also the quotation in (i); Walton 2001, 164).

(iv) The partial basis of plausible inferences. With deductive inferences, the premises make up a 'full basis' in the sense that "[i]f we receive some new information that does not change our belief in the premises, it cannot change our belief in the conclusion" (Polya 1948, 223). On the other hand, in the case of plausible inferences the premises make up only a 'partial basis', that is the complete basis has a part which is not expressed through the premises:

"[...] the premises constitute only one part of the basis on which the conclusion rests, the fully expressed, the 'visible' part of the basis; there is an unexpressed, invisible part, formed by something else, by inarticulate feelings perhaps, or by unstated reasons. In fact, it can happen that we receive some new information that leaves our belief in both premises completely intact, but influences the trust we put in $A$ in a way just opposite to that expressed in the conclusion. To find $A$ more plausible on the ground of the premises of our heuristic syllogism is only reasonable. Yet tomorrow I may find grounds, not interfering at all with these premises, that make $A$ appear less plausible, or even definitively refute it. The conclusion may be shaken and even overturned completely by commotions in the invisible parts of its foundation, although the premises, the visible part, stand quite firm." (Polya 1948, 223f; emphasis added) 
(v) The context-dependence of plausible inferences. In plausible inferences the conclusion cannot be detached from the premises. Moreover, Polya emphasizes that the 'weight' of the conclusion depends not only on the premises explicitly formulated, but also on hidden grounds which form the 'invisible' part of the partial basis such as the individual's background or cultural knowledge, etc. (Polya 1954, 115f). The conclusion of a plausible inference is always only provisional; its acceptability is closely dependent on the particular circumstances given. Rescher maintains, too, that it is definitely not universalizable formal considerations that determine the evaluation of the plausibility of conclusions, but rather, it is clearly situation-specific, contextual factors in the widest sense (Rescher 1976, 111ff).

(vi) The plausibility of the premises and the emergence of contradictions. It is one of the basic issues of plausibility theory that it can treat both the emergence and the resolution of contradictions.

Firstly, those premises which we are compelled to treat as the starting point of our reasoning are not to be regarded as certainly true, but can only be assumed to be plausible in the given context, that is, they are more credible than their potential alternatives if certain conditions are satisfied. This, in accordance with the nature of the partial basis mentioned in (iv), may result in the inconsistency of the basis which we have to use as our background for reasoning. In this sense, the basis is informationally overdetermined. We have to reason by making use of not completely trustworthy information - that is, building on a partial basis. We know that only plausible inferences can be drawn from a partial basis, and these inferences can lead to contradictory conclusions in certain cases: to explain one's data, one may set up hypotheses which mutually exclude each other but which are plausible in certain respects in a given context (Rescher-Brandom 1979, 160). That is, the emergence of contradictions may have its sources in plausible inferences. ${ }^{10}$

Secondly, resolving this kind of informational overdeterminacy is only possible if we decide what to abandon amidst bits of information which contradict each other. To achieve this, we need to exceed purely formal considerations, as was argued for in point (v), and somehow have to compare the possible alternative decisions. If we consider conclusions to be tools in information processing (for more on this, cf. Rescher 1976, 97ff),

${ }^{10}$ Of course, a partial basis does not necessarily lead to the emergence of contradictions, but the emergence of contradictions is one possibility that may arise from the peculiarities of the partial basis.

Acta Linguistica Hungarica 52, 2005 
plausible inferences can be perceived as converting a set of premises into information of a certain plausibility, that is into a conclusion (cf. also point (vi)). This provides an opportunity for us to compare the conclusions that can be drawn from particular subsets of an informational set and choose the one which appears to be the most probable, the most optimal, and the most credible for us. One may use plausible inferences once again to resolve the (possible) contradictions that emerge as the conclusions of plausible inferences drawn from the partial basis, examining which is the most credible of the alternatives in a given context-hoping that sooner or later an informational state is reached wherein novel contradictions do not arise. That is: one of the possible means of resolving contradictions is plausible inference.

To sum up: the simplest way to characterize the strong correlation between plausible inferences and the emergence of contradictions is to regard plausible inferences as one of the possible sources of the emergence of contradictions on the one hand, and as one of the possible means to resolve contradictions on the other.

(vii) The cyclic and prismatic nature of plausible reasoning. We reason cyclically by starting off from an inconsistent set of premises. We return to the problems in question again and again, and supplementing the partial basis with different latent background assumptions we transform the set of information at our disposal by drawing additional plausible inferences, and re-evaluate the credibility of the respective data (hypotheses, alternative explanations). During these cyclic returns we aim to filter out hypotheses unacceptable for some reason gradually, according to different - possibly contradictory - considerations (Rescher $1987,304 ; 1976,111 \mathrm{ff}, 118) .{ }^{11}$ This way it becomes possible to compare one's cycles and to assess one's progress. First and foremost, there are two questions one may consider during this process:

(a) The first question is whether one has managed to root out the contradictions within a particular cycle (that is whether one has gained a consistent set of information), or whether at least the plausibility of any of the contradictory hypotheses has increased.

${ }^{11}$ See Kertész-Rákosi (2005a;b) considerably more detailed characterizations of the cyclic nature of argumentation in linguistics supported by a series of case studies. 
(b) The second question concerns the degree of plausibility of the complete amount of information within a reasoning cycle as compared to the total of other reasoning cycles. ${ }^{12}$

Moreover, the cyclic reasoning is at the same time prismatic as well. The relation between the cyclic and the prismatic nature of reasoning is formulated by Rescher clearly:

"[...] it may make perfectly good sense to proceed dialectically and consider an issue prismatically, by proceeding in the variable light of not merely different but even inconsistent perspectives."

(Rescher 1987, 306f; emphasis added)

"[...] 'dialectical' reasoning is a matter of the repeated reconsideration of old issues from newly attained points of view. The root idea of such reasoning is that of a multistage process where we repeatedly re-examine one selfsame issue from different and mutually inconsistent points of view. It is a matter of developing a course of reasoning in several phases or 'moments' where we consider an issue now from different and mutually incompatible points of departure, moving in a round-about way from $\mathrm{P}$ via non-P to a conclusion of some sort. We proceed in circles or cycles where we return to a certain issue now in this light and now in that. We do not constantly press onwards to new ground, repeatedly crisscrossing the same terrain, approaching the old issues from different and often discordant angles. [...] In dialectical reasoning we make assertions that are negated ('corrected' so to speak) by subsequent counter-assertions. We have here a process of successive approximation as it were, where at each stage we assert things that are literally false and in need of eventual correction. When things go smoothly, however, these successive corrections appertain to increasingly minor and insignificant respects. [...] At each stage what we say is not literally correct but only correct up to a point." (op.cit., 303f; emphasis added)

(viii) The universality of plausible inferences. Although Polya discusses the mechanisms of plausible inference mainly in the domains of

12 This cyclic nature of reasoning has to be clearly distinguished from circularity: "This circular process highlights the element of 'self-correction' present in systematic applications of plausibility analysis, allowing for a revised appraisal of the initial data that provide the very materials of the analysis. There is a cyclic movement, a closing of the cycle which requires a suitable meshing - a matching process that eventually retrovalidates (i.e., retrospectively revalidates) the criteria of plausibility assessment with reference to the results to which they lead. [...] The sort of 'self-criticism' at issue does not reflect any vicious or vitiating circularity, but in effect amounts simply to a feedback process that uses later, more refined stages of the analysis to effect revisionary sophistications in the materials from which earlier stages proceeded. One indeed returns to 'the same point' but does so at a different cognitive level." (Rescher 1976, 119; emphasis as in the original and added)

Acta Linguistica Hungarica 52, 2005 
mathematics and the sciences, he also emphasizes that these mechanisms are universal and not specific to these domains:

\begin{abstract}
"The underlying principle is generally recognized in the natural sciences, and it is implicitly admitted in the law courts, and in everyday life. The verification of a consequence is regarded as reasonable evidence for a conjecture in any domain. Thus our patterns appear as universal.

We pay, however, a price for such 'universality'. Our pattern succeeds in being universal because it is one-sided, restricted to one aspect of plausible inference. The universality becomes blurred when we raise the question 'What is the weight of such evidence?' In order to judge the weight of the evidence, you have to be familiar with the domain; in order to judge the weight with assurance, you have to be an expert in the domain. Yet you cannot be familiar with all domains, and you can still less be an expert in all domains. And so everyone of us will notice soon enough that there are practical limits to the universality of plausible inference." (Polya 1954, 114; emphasis added)
\end{abstract}

One important consequence of this stance is that scientific and everyday reasoning may rest on the same patterns of inference, ${ }^{13}$ although, of course, from domain to domain these patterns may be manifested in very different ways. Thus, at the outset nothing speaks against our assumption that even the cognitive base of pragmatic principles may be governed by plausible inferences rather than probabilistic associations in the sense of Robinson. Before, however, elaborating on this, let us turn to an issue which is of utmost importance. In particular, $\left(\mathrm{S}^{\prime}\right)$ cannot be maintained if the patterns of plausible inference are considered to be merely abstract constructs which are intended to model how people argue. Therefore, in the next section we will show that the cognitive reality of plausible inferences may be justifiedly hypothesized, although we know very little about these cognitive processes and such a hypothesis is far from having been proved convincingly.

\title{
2.2. On the cognitive reality of plausible reasoning
}

We do not claim that the mind is a plausibilistic system. However, we assume two things. Firstly, that the cognitive mechanisms we try to capture 'look like' plausible inferences. Secondly, that the relations which we

${ }^{13}$ Our hypotheses $\left(\mathrm{S}^{\prime}\right)$ and $\left(\mathrm{S} 2^{\prime}\right)$ are closely related, because we assume that both scientific reasoning and everyday communicative behaviour make extensive use of plausible inferences. See sections $\mathbf{4}$ and $\mathbf{5}$.

Acta Linguistica Hungarica 52, 2005 
interpret as plausible inferences may have some kind of cognitive reality, although we do not know exactly what neural processes are at work. ${ }^{14}$ Let us, without striving for a comprehensive discussion of the literature, mention two instructive examples illustrating the psychological aspects of reasoning in general.

Dolinina (2001) examined the use of 'theoretical' ('logical', 'formal', 'deductive') and 'empirical' ('pre-logical', 'traditional', 'inductive') inferences on the basis of neuropsychological evidence. She proposed the following solution to what is called the problem of the heterogeneity of thinking:

\begin{abstract}
"[...] neurological experiments demonstrated that both mechanisms of reasoning are simultaneously present in the brain of one and the same person, that both of them can be used, but that each of them is controlled by a different hemisphere. [...] these results give a certain counterevidence to Johnson-Laird's claim that formal reasoning is not represented in the mind. [...] In the case of reasoning patterns, the right hemisphere appears to control the quality of information (e.g., the truthfulness of premises, testing them against the realities of the world and/or personal knowledge/ experience), whereas the left hemisphere is responsible for the correctness of purely operational mechanisms (formal correctness of inferences). [...] Since literate western-schooled individuals possess both modes of reasoning, the question was raised [...], which of the modes is normally used [...]. Some cognitive psychologists (e.g., Johnson-Laird and Moore) claim that the traditional, semantic way of reasoning is responsible for reasoning processes and is represented in the mind, the formal being only a 'performance' strategy. Others (Wilson and Sperber) stress the priority of formal reasoning. Deglin's neurological experiments on functional differentiation of right and left hemispheres demonstrated that both strategies are present in the brain [...], so an individual can choose whatever strategy is most appropriate to the circumstances." (Dolinina 2001, 130ff)
\end{abstract}

The neurological experiments carried out by Dolinina support the assumption that there exist inferential patterns represented in the mind. However, the evidence she refers to is not sufficient for giving a sophisticated and convincing answer to the question of what kind of structure

${ }^{14}$ This cautious claim of ours is analogous to Robinson's evaluation of his own approach: "The point [...] is not to say that the mind is a probabilistic system. What it actually is, at yet another level of abstraction, is a bunch of interconnected neurons reacting to the world. But the overall behaviour created by a neural system situated in an environment looks like what we describe as probabilistic behaviour. This behaviour, described best with a cognitive model such as the one outlined above, can result in the kinds of behaviour described by linguistic models of pragmatics at their level of description." (Robinson 1997, 264; emphasis added)

Acta Linguistica Hungarica 52, 2005 
these patterns of inference have and of what the application of particular patterns normally depends on.

She hypothesizes that if one is able to judge the truth value of the premises, then one prefers the 'empirical' mode, i.e., the use of the right hemisphere of the brain, whereas with respect to domains one is not familiar with, the 'logical' mode, i.e., the use of the left hemisphere is preferred. That is, she assumes a model which isolates the use of inferential patterns from the content of the premises.

However, in many cases where the 'empirical' mode was used, the reasons given for the responses contained inferences as well, even if the left hemisphere was suppressed or the experimentee had no formal education. Then the task of the right hemisphere might be not to provide the structure of inferences in general and the latter cannot be identical with the rules of formal logic. Thus, the difference between the two 'reasoning strategies' seems to lie not in the use of reasoning patterns but rather, in the consideration of the content of the premises. Accordingly, the 'theoretical reasoning mode' can be characterized as the ability to think hypothetically on the basis of formal rules of deductive logic.

The answers described by Dolinina as examples of the 'empirical' reasoning mode can be accounted for with the help of the theory of plausible reasoning. Since, according to Polya (1954, 42ff), patterns of plausible reasoning can be seen as tending to the corresponding pattern of demonstrative inference when the credibility of the premises tends to certainty, the mechanisms of plausible reasoning might explain the use of seemingly deductive inferences by uneducated persons or by experimentees whose left hemisphere was suppressed, too.

As a second example, we refer to research carried out by Politzer and Bourmaud (2002) who describe experiments which tried to give an answer to the question of what conclusions can be drawn if in inferences of the type 'modus ponens' or 'modus tollens' the truth of the 'if ... then' premise is uncertain or if there is at least another premise which undermines the truth of the major premise. They observed that the experimentees judged the uncertainty of inferences much higher with respect to medical topics than, for example, topics concerning mechanics. That is, Politzer and Bourmaud, too, consider the application of inferences to be domain-dependent, but they characterize the sources of this domain-dependence in a more sophisticated way than Dolinina does:

"The key factor seems to be the awareness that the level of understanding of
the causal link between antecedent and consequent of the conditional differs 
from one domain to another: for the participants, the link may be more or less strong, allowing for more exceptions to the hypothesis if it is weaker." (Politzer-Bourmaud 2002, 347; emphasis added)

"[...] there is one single common mechanism, namely the recognition of one or several factors that are necessary conditions for the consequent to occur and, by this very fact, are conditions that implicitly complement the antecedent of the conditional to make it an actual sufficient condition. The degree of belief in the satisfaction of those factors acts as a mediator to define the degree of sufficiency of the conditional premise, that is, its credibility, and consequently, by inheritance, the degree of belief in the conclusion of the argument. The truth status of the conclusion is treated by degree rather than in all-or-nothing manner, and this degree is closely correlated to the degree of belief in the premise." (Politzer-Bourmaud 2002, 353; emphasis added)

As opposed to Dolinina's hypothesis, Politzer and Bourmaud's findings speak for the cooperation of the formal aspect and the content. These findings are in full accordance with Polya's claim that the strength of the conclusion is directly proportional to the strength of the premises and that normally inferences rest on a partial basis (see 2.1 (iv), (v), and Polya 1954, 41ff).

These two examples witness that, on the one hand, it is reasonable to assume that plausible inferences are not merely abstract theoretical constructs and that they have some kind of cognitive reality; nevertheless, on the other hand, research in this field is still immature and we know very little about the details of this cognitive reality.

In the next two sections we will illustrate how our model can capture some of the issues raised in section $\mathbf{1}$.

\section{On $\left(\mathrm{S2}^{\prime}\right)$}

\subsection{On the correlation between plausible inferences and the cognitive basis of pragmatic principles}

So as to show the capability of our approach to plausible reasoning to capture the problem (P2), first we have to demonstrate that the main tenets of this approach correlate with those aspects of the cognitive base of pragmatic principles which Robinson points out.

As Robinson $(1997,262)$ maintains, one of the difficulties which one encounters in trying to analyse an example like

(3) I am looking for a doctor.

Acta Linguistica Hungarica 52, 2005 
is that although it is assumed that the speaker means a medical doctor, there is an infinite amount of background information that may specify the meaning of doctor in different ways. For example, at a dissertation defence party, probably a person with a PhD degree may be meant. Now, objective theories in the sense of section $\mathbf{1 . 1}$ are expected to clarify the way particular contexts cause particular interpretations, whereas this is, as we have seen, not possible.

Robinson suggests a solution to the problem of accounting for the infinity of the background information yielding the possible interpretations of (3) which centers on the idea of a distributed system that makes use of probabilistically stored concepts grounded relative to the environment. He presumes that "related things are probabilistically associated together in terms of, and reflecting the strength of, the frequency of their associations in the world" (Robinson 1997, 262). Thus, concepts are assumed "to generalize statistically towards a statistically defined centre, or centres, at the same time that their associative nature automatically results in context-sensitive activation" (op.cit., 263).

Alternatively, we assume that the interpretations of terms like doctor in (3) emerge as the result of drawing plausible inferences from a partial basis in the sense of (iv) in section 2.1. This means, firstly, that on the one hand the infinity of the basis is acknowledged, on the other hand, however, it is also acknowledged that the only part of this basis accessible to the hearer, i.e., what he/she encounters is a partial basis. Whereas Robinson assumes a probabilistic and non-inferential mechanism, we hypothesize a plausibilistic and inferential one. Revealing the mechanisms according to which plausible inferences are drawn from a partial basis is one of the major achievements of plausibility theory.

The mechanism of drawing plausible inferences from a partial basis is, as we emphasized in (v) in section 2.1, deeply context-dependent. The fact that plausible inferences are context-dependent is important, because context-dependence is one of the major features of the cognitive basis of pragmatic principles:

\footnotetext{
"[...] there are an infinite number of possible contexts for each utterance, so we are faced with the problem discussed earlier about objective systems, needing to specify the full base of understanding of an utterance; everything must be represented or derivable from a representation."

(Robinson 1997, 262; emphasis added)
}

Therefore, in principle plausibility theory is capable of capturing this aspect of the basis at the outset. 
Another relevant feature of plausible inferences is that they are closely connected both to the emergence and the resolution of inconsistencies. Since, as we have seen in (vi) in section 2.1, the conclusion of plausible inferences is not true with certainty, but only more or less credible; new information may turn up any time and can undermine our trust in the conclusions, compelling us to reconsider the situation at issue and to revise both the premises and the inferences from them. It is one of the most attractive impacts of plausibility theory that it is capable of accounting for these mechanisms (see Kertész 2004b, chapter 22; Kertész-Rákosi 2005a and Rákosi 2005 for detailed discussion). Now, the emergence of inconsistencies is one important property of the cognitive basis of pragmatic principles as well:

\footnotetext{
"If something in later discourse or activity should appear to contradict or modify this specific understanding, then a modification of the active understanding is possible; one is only adding information and not contradicting a previous assumption." (Robinson 1997, 266; emphasis added)
}

Thus, we may maintain that in principle plausibility theory is appropriate for analysing the emergence and resolution of contradictions in the cognitive basis of pragmatic principles.

Finally, in section $\mathbf{1 . 3}$ we concluded among other things that it is not unreasonable to assume that with respect to the phenomena Robinson's considerations focus on there is a kind of cyclic and prismatic inference and decision process at work. In (vii) in section $\mathbf{2 . 1}$ we demonstrated that it is precisely cyclic and prismatic procedures that are at the heart of plausible inferences. Therefore, it is only natural that there may be a kind of correspondence between the mechanisms governing plausible inferences and the prismatic and cyclic relations between pieces of information that constitute the cognitive basis of pragmatic principles.

After having pointed out the appropriateness of plausibility theory for suggesting a possible solution to (P2), what remains to be shown is how plausibility analysis works. So, the next section will be devoted to the analysis of instructive examples.

\subsection{Examples}

\subsubsection{First example}

(4) John: Did you go to Bloomingdale's when you went to New York? Mary: Well, what do you think of my new dress? (cf. Robinson 1997, 257) 
Cycle 1. The information at John's disposal is insufficient, because he does not know whether Mary was at Bloomingdale's or not. Therefore, John tries to get rid of the informational underdetermination. On the basis of his cultural knowledge he knows that Bloomingdale's is a department store in New York.

Nevertheless, there is a piece of information which indicates that the answer might be yes: he understands that Mary was in New York where this department store is situated. Thus, a necessary condition ${ }^{15}$ for the affirmative answer is probably satisfied:

(5) 〈If Mary went to Bloomingdale's, then she was in New York. $\rangle^{16}$

It is possible that Mary was in New York.

After it has become more credible that Mary was in New York (since John understood so and Mary did not protest against this presupposition of John's question), it is more credible that Mary was at Bloomingdale's.

This inference is an instance of shaded reduction. ${ }^{17}$

Of course, this is only one of several possible arguments which John uses to reach conclusions. As a first approximation, Mary's answer leaves the informational underdetermination of the partial basis untouched. It facilitates drawing further inferences by providing John with a new piece of information. That is, the partial basis which John's inferences rest on are supplemented by the new information that Mary has a new dress.

Cycle 2. John evaluates the situation from another perspective in that he considers the information content of Mary's answer. He attempts to supplement the partial basis by further background information. At this point associations may play a significant role; that is, he has to find cultural knowledge which could be used in the situation at issue. For example:

15 That Mary was in New York is a necessary condition for her having visited Bloomingdale's, because she could not have been in this department store if she had not gone to New York.

${ }^{16}$ In our analyses " $\langle\ldots\rangle$ " will indicate implicit premises.

${ }^{17}$ For lack of space, we cannot introduce the patterns of plausible reasoning systematically and elucidate the patterns we will apply in the examples to follow. Let it be sufficient to refer to Polya (1954); Kertész (2004b); Rákosi (2005). For the reader's convenience, however, in the appendix we enumerate the patterns which we make use of in the analyses. 
(6) (It is possible that if Mary has a new dress, then she bought it. ${ }^{18}$

Mary has a new dress.

It is more credible that she bought it.

This inference is an instance of shaded modus ponens.

John's cultural knowledge also includes the information that Bloomingdale's is a department store which sells dresses. This information gives rise to an analogical inference yielding the conclusion that Mary could buy a dress there, too:

(7) (It is possible that if Mary bought a new dress, then she did this at Bloomingdale's. $\rangle$

It is possible that she bought a new dress.

After it has become more credible that Mary bought a new dress (cf. the previous inference), it is more credible that Mary did this at Bloomingdale's.

This inference is an instance of shaded modus ponens.

Each of the above inferences rest on uncertain premises, therefore they yield conclusions whose truth value is uncertain, too. As a result of the peculiarities of the partial basis mentioned in section 2.1, this means that new information may turn up which contradicts the conclusions already drawn. However, at this point John does not possess information of the latter kind.

However, it is not difficult to construct situations in which he does. For example, the context-dependence of implicatures is indicated by the fact that if John knows that Mary never buys dresses at Bloomingdale's because the only thing she buys there is caviar, then he will draw the opposite conclusion. That is, in this case the last inference of cycle 2 will be as follows:

(8) 〈It is possible that if Mary visited Bloomingdale's, then she did not buy a dress. It is possible that Mary did not buy a dress.

After it has become less credible that Mary did not buy a dress, it is less credible that Mary visited Bloomingdale's.

This inference is an instance of shaded modus tollens.

At this point John's knowledge includes an inconsistent set of assumptions, because the result of cycle 1 contradicts the result of cycle 2 .

${ }^{18}$ Of course, we cannot exclude that she made the dress herself or that it was presented to her by her husband. That is why it is only possible that she bought it.

Acta Linguistica Hungarica 52, 2005 
Cycle 3. So as to resolve the contradiction, John has to decide which conclusion he thinks to be more plausible. Accordingly, he examines the problem from another perspective:

(9) 〈It is possible that if Mary visited Bloomingdale's, then she bought caviar. ${ }^{19}$

It is possible that Mary bought caviar.

After it has become less credible that Mary bought caviar (because she did not mention it), it is less credible that Mary went to Bloomingdale's.

(9) is an instance of shaded modus tollens.

The last conclusion would speak for the assumption that Mary did not visit Bloomingdale's. Nevertheless, it is also possible that John reconsiders a certain part of his background knowledge:

(10) (It is possible that if Mary bought a new dress, then she did this exceptionally at Bloomingdale's.

It is possible that Mary bought a new dress.

After it has become more credible that Mary bought a new dress, it is more credible that she bought it exceptionally at Bloomingdale's.

(10) is an instance of shaded modus ponens.

However, it is not at all certain that John can decide between the above alternatives on the basis of the information at his disposal; it may be the case that he will ask further questions.

Of course, (5)-(10) are only some of the possible reconstructions of (4). Nevertheless, they have illustrated how implicatures can be accounted for by plausibility analysis.

\subsubsection{Second example}

It is worth considering what happens when a third person, say Jim, who does not know anything about department stores in New York, attends the dialogue. Probably he will start his inferences by considering Mary's answer:

(11) (It is possible that if Mary has a new dress, then she bought it.)

Mary has a new dress.

It is more credible that she bought it.

19 This premise is the result of an analogical inference.

Acta Linguistica Hungarica 52, 2005 
(11) is an instance of shaded modus ponens.

Then he tries to find a connection between New York and the purchase of the new dress:

(12) 〈It is possible that if Mary bought a new dress, then she bought it in a department store in New York.)

It is possible that Mary bought a new dress.

After it has become more credible that she bought a new dress, it is more credible that she bought it in a department store in New York.

(12) is an instance of shaded modus ponens.

(13) It is possible that Bloomingdale's is in New York. ${ }^{20}$

It is possible that the department store in which Mary bought her new dress is in New York.

It is possible that Mary bought her new dress at Bloomingdale's.

(13) is an analogical inference.

The inferences which Jim carried out are even less certain than those carried out by John, and accordingly, the plausibility of the conclusions Jim has drawn are less plausible than those drawn by John.

\subsubsection{Third example}

The Gricean theory explains the following example by the violation of the maxim of relevance:

(14) Peter: Mrs. Johnson is an old hag.

Rose: The weather was beautiful this summer, wasn't it?

We will show that this phenomenon can be explained with the help of our model in the following way.

Cycle 1. First of all, Peter tries to relate his own utterance and Rose's reply. However great his efforts are, he does not succeed in finding a chain of inferences in which these two pieces of information work as premises. Therefore, he notices that Rose's reply is irrelevant. ${ }^{21}$

${ }^{20} \mathrm{Jim}$ can infer this information from the fact that it is presupposed by John's question. Cf. cycle 1 in 3.2.1.

${ }^{21}$ Accordingly, here 'relevance' means that on the basis of the utterance and the information given, one is able to construct a chain of inferences which rule out the informational under- or overdeterminacy; otherwise the utterance is irrelevant. 
Cycle 2. It may be the case that Peter, due to this conclusion (and perhaps due to the awkward silence accompanied by Rose's unsympathetic face), is so embarrassed that he puts up with not understanding Rose's reaction and with the fact that he got entangled in such an awkward situation. However, if he has already been in a similar situation, then he may draw an analogical inference which will yield the implicature of Rose's utterance:

(15) When Betty answered Bruno in an irrelevant way, then Bruno's utterance touched on something unpleasant for the hearers who did not want to speak about this topic.)

Rose answered me in an irrelevant way.

It is possible that I said something which touched on something unpleasant for the hearers.

(15) is an analogical inference.

It may also be the case that Peter realizes this conclusion by considering Rose's gestures and the sudden awkward silence. This makes him understand that irrelevance indicates the rejection of the topic:

(16) 〈When Betty's answer was preceded by an awkward silence and other people also looked disapprovingly at Bruno, then they did not want to speak about the topic Bruno had just touched on. $\rangle$

Rose's answer was preceded by an awkward silence and also others looked disapprovingly at me.

It is possible that I said something which was embarrassing for the others and they did not want to speak about the topic at issue.

(16) is an analogical inference.

(17) (It is possible that when Bruno said something which was embarrassing for the others, and they did not want to speak about the topic at issue, then Betty answered in an irrelevant way, because she wanted to make Bruno notice this.)

It is possible that I said something which was embarrassing for the others, and they did not want to speak about the topic at issue.

It is possible that Rose answered me in an irrelevant way because she wanted to make me notice this.

(17) is an analogical inference.

The inferences also show that after the first cycle Peter changed the perspective from which he evaluated the situation. 


\title{
3.2.4. Fourth example
}

Levinson (2000) discusses, among other things, basic properties of generalized implicatures. He, too-like Robinson-claims that formal logic cannot serve as a tool for the reconstruction of implicatures. However, unlike Robinson and in accordance with our hypothesis, he concludes that implicatures can be explained by reconstructing them as the results of plausible inferences:

\begin{abstract}
"There is a [...] problem that has received less attention, what might be called the logical problem of reconstructing speaker's intentions [...]. Let us (following Aristotle) assume that we reason from goals to actions utilizing a logic of action, or a practical reasoning. Now, some theorists have assumed that intention-recognition is simply a matter of running that reasoning backwards [...]: we observe the behavior and figure out the underlying intention by the same rules that we convert intentions into the actions that will effectuate them. The logical problem is that this cannot work, for the simple reason that for all inference systems one cannot work backwards from a conclusion to the premises from which it was deduced - there is always an infinite set of premises which might yield the same conclusion [...]." (Levinson 2000, 30; emphasis added)
\end{abstract}

"What [we] clearly need is a set of heuristics, mutually assumed by sender and receiver, that can serve to multiply the coded information by a factor of, say, three, by licensing inferential enrichment of what is actually encoded by choice of a specific signal. These heuristics must at the same time constrain that enrichment in such a way that the overall message can still be correctly recovered, by guiding (or coordinating) the match between the chosen signal and the recoverable, augmented message." (op.cit., 30f; emphasis added)

According to Levinson, these heuristics rest on a special kind of plausible reasoning, namely default logic:

"GCIs [generalized conversational implicatures] are inferences that appear to go through in the absence of information to the contrary; but additional information to the contrary may be quite sufficient to cause them to evaporate. Thus the mode of inference appears to have two important properties: it is a default mode of reasoning, and it is defeasible. [...] A reasoning system is said to be defeasible (or when instantiated in an argument nonmonotonic) if an inference or argument in that system may be defeated by the addition of further premises. [...] Default logics aim to capture a [...] mode of reasoning - namely, the notion of a reasonable presumption, a ceteris paribus assumption." (ibid., 42ff; emphasis as in the original)

Nevertheless, besides similarities, there are also crucial differences between our and Levinson's approach. The aim of the following brief anal- 
ysis is only to illustrate the difference between our solution and Levinson's; the scope of the present paper does not permit a more extensive treatment of these differences. ${ }^{22}$ For example,

(18) At our university some professors go prepared to their lectures.

implicates that

(19) At our university not all professors go prepared to their lectures.

Cycle 1. The phrase some $X s$ are $Y s$ means that there are $\mathrm{Xs}$ which are Ys:

(20) There are professors at our university who go prepared to their lectures.

However, some $X s$ are $Y s$ does not say anything about whether all Xs are Ys; its information content leaves both possibilities open.

Cycle 2. If the hearer wants to do away with the informational underdetermination for some reason, ${ }^{23}$ he or she has to examine the situation from another perspective. The following consideration presents itself immediately:

${ }^{22}$ It is interesting that the example we chose seems to refute Levinson's claim that default conclusions are cancelled if contradicting information is made available.

${ }^{23}$ Experiments with children show that we need some reason to go beyond the literal meaning of the utterances: "If preschoolers, unlike adults, cannot readily infer the pragmatic nature of the task, and are not given adequate motivation to go beyond the truth conditional content of the utterance, they may readily settle for a statement which is true but does not satisfy the adult expectations of relevance and informativeness." (Papafragou-Musolino 2003, 269)

For adults the use of certain phrases, i.e., knowledge of language may fulfil this task (for example some, start). But the presence of such phrases is not a necessary condition for implicatures: "[...] scalar inferences can be induced by partial contextual orderings, which may be supplied by stable world knowledge or created in a completely ad hoc fashion [...]:

A: Did you get Paul Newman's autograph?

B: I got Joanne Woodward's.

$\rightarrow$ B did not get Paul Newman's autograph." (Papafragou-Musolino 2003, 258)

Acta Linguistica Hungarica 52, 2005 
(21) (It is possible that if there were no professor who would go unprepared to his lectures, ${ }^{24}$ then the speaker would have said that At our university all professors go prepared to their lectures.)

The speaker did not say that At our university all professors go prepared to their lectures.

It is less credible that there is no professor who goes unprepared to his lectures (i.e., it is more credible that there are professors who go unprepared to their lectures).

This inference is an instance of shaded modus tollens.

Cycle 3. Our cultural knowledge also contains information which contradicts this conclusion. Namely, one of the prototypical features of professors is that they go prepared to their lectures:

(22) Professors go prepared to their lectures.

The conclusion obtained within cycle 2 and (22) results in inconsistency which has to be resolved by the hearer somehow.

Cycle 4. (22) is an element of the speaker's cultural knowledge as well. ${ }^{25}$ Despite this, he or she has used some instead of all in (18). So, it is possible that he or she does not agree with (22).

(23) /It is possible that if the speaker had agreed with the statement that At our university all professors go prepared to their lectures, he or she would have uttered this.)

The speaker did not say that At our university all professors go prepared to their lectures.

It is less credible that the speaker agrees with the statement.

(23) is an instance of shaded modus tollens.

This is an argument for the implicature mentioned in (19). The hearer's task, then, is to consider the strength of the arguments for and against the implicature.

\subsubsection{Summary}

The analyses of the examples were intended to illustrate that it is possible to explain different kinds of implicatures by making use of plausibility

\footnotetext{
${ }^{24}$ I.e., if all professors go prepared to their lectures.

${ }^{25}$ This is an analogical inference.
}

Acta Linguistica Hungarica 52, 2005 
analysis. The analyses have shown that implicatures can be assumed to work along the same lines as plausible inferences do. That is, they are characterized by

(a) drawing inferences from a partial basis,

(b) using different patterns of plausible inferences,

(c) the emergence and resolution of contradictions,

(d) context-dependency,

(e) the cyclic and prismatic revaluation of the information available.

\section{On $\left(\mathrm{S}^{\prime}\right)$}

In section 1.1 we raised two closely related problems: a metascientific and an objectscientific one. Up to this point we tried to solve the objectscientific problem (P2) by the application of plausibility analysis. Nevertheless, the method of our scientific method, namely, plausibility analysis is no less problematic than the nature of implicatures: our findings depend on the effectivity of this method to a considerable extent. Therefore, it is important to reflect on our own argumentation metascientifically so as to clarify some of the methodological difficulties we encounter:

(a) Our own line of reasoning started from uncertain premises as well: since we had no convincing evidence at our disposal, we assumed only hypothetically that the inferences we discussed in the previous section were or could have been carried out.

(b) This means that there was only a partial basis which our inferences could rely on.

(c) Not only the persons in the above examples, but we, too, as researchers had to struggle with the informational underdetermination of the partial basis.

(d) The way we applied plausibility analysis is only one side of the prism through which we observed the object of our investigations, namely, the cognitive basis of pragmatic principles.

(e) Accordingly, we ourselves as analysts had to proceed cyclically and prismatically by making use of the perspectives of cognitive psychology and linguistics, and above all of our own cultural knowledge. For example, as we have seen, particular aspects of the theory of associations which Robinson argued for could be integrated with our approach, although in a somewhat reinterpreted way: they may give an answer to the question of how the premises which contribute to filling the informational gap in the partial basis are selected. 
In what follows, we will make a few brief remarks on how this kind of cyclic and prismatic reasoning works with respect to the construction of pragmatic theories. Accordingly, we will motivate our hypothesis $\left(\mathrm{S}^{\prime}\right)$ as a possible solution to (P1). However, we will not go into a detailed discussion, because elsewhere we put forward possible applications of plausibility analysis to theory construction in linguistics. ${ }^{26}$

As we saw in section 1.1, Robinson claims that we must depart from a theory of cognition and proceed toward a descriptive theory of linguistic behaviour. The solution proposed by Robinson to (P1) consists in assuming a hierarchy of systems where every level is determined by a lower level. These different, hierarchically related systems correspond to different theories. ${ }^{27}$

Accordingly, in Robinson's view, the relationship between the objectand the meta-level is always unidirectional. However, we showed in section $\mathbf{1 . 3}$ that this methodology inevitably runs into an infinite regress which can be interrupted only by stopping at the level at which there is no avoiding the identification of the structures of the theoretical description with structures of the mind. Moreover, we also saw that the researcher uses his/her own cultural knowledge as a point of departure and projects it onto a lower level system. Now, the question arises whether our approach results in the same problems, namely, those summarized in $(\mathrm{P} 1)(\mathrm{a})-(\mathrm{d})$.

To begin with, let us mention that Hample (1985/1992) - in referring to O'Keefe (1977/1992) - differentiates between three perspectives of investigating argumentation. Argument ${ }_{1}$ examines the products of reasoning; it handles inferences as being static representations of reports

\footnotetext{
${ }^{26}$ See Kertész (2004a;b); Kertész - Rákosi (2005a); Rákosi (2005).

27 "I have attempted to show how regularities in one theory may be emergent from the behaviour of a lower-level system. [...] By relating this model to the regularities described at a linguistic level of behaviour we do avoid the problems created by directly instantiating these linguistic models as cognitive theories. But, because this model is also a description, we are left with the question of whether or not we will run into the same problems as before, only at a different level of description. Unfortunately, the answer here may well be yes. It is therefore necessary to take the relationship between the two levels of modelling I have discussed one step further. [...] In this view, we must use our objective theories not as ends in themselves but as methodological tools to point to regularities in one system as emergent from another. [...] This means we will need multiple levels of description capturing all of the situational, behavioural, and biological phenomena that interact to create organized behaviour. None of these, in isolation, will be an adequate model of cognition." (Robinson 1997, 268f; emphasis added)
} 
on the results of drawing inferences. It suggests that the aim of argumentation theory is the analysis of such reports. The paradigm example of this view is formal logic. ${ }^{28}$ Seen from the point of view of argument $_{2}$, argumentation is a process which aims at influencing the hearer's beliefs or actions. In this respect, argumentation theory should focus on revealing the factors underlying argumentation as interaction. The third view, i.e., argument $_{0}$, centers on the mental processes governing the construction of arguments. It seeks to find out to what extent and in what way mental processes are constitutive of the peculiarities of arguments. Thus, assuming that argumentation in general and inferences in particular have some kind of mental reality is one of the hypotheses widely discussed in the contemporary literature of argumentation theory.

Hample introduces a further distinction, namely that between weak and strong claims for argument $_{\mathbf{0}}$. The weak view focuses on argument ${ }_{1}$ or argument $_{2}$ and attributes less relevance to the cognitive aspects of argumentation. It suggests a methodology which takes the opposite direction to Robinson's proposal: we should choose the investigation of argument ${ }_{1}$ or argument ${ }_{2}$ as our starting point and proceed towards argument ${ }_{0}$, because the structure of argument ${ }_{0}$ can be entirely reconstructed from the investigation of $\operatorname{argument}_{1}$ or argument $_{2}$. Thus, we can construct a theory of argument $_{0}$ if we proceed from the theory of argument ${ }_{1}$ or argument ${ }_{2}$ towards a theory of argument $_{0}$. It is clear that this attitude is similar to the point of view of the pragmatic theories mentioned in this paper.

Strong claims for argument $_{0}$ (which, according to Hample, are rarely encountered) take the opposite direction: they try to understand argumentation by starting from the mental and proceeding towards its public manifestations. Argumentation in this sense is not something special, because it involves a wide variety of cognitive events such as perceiving, remembering, associating, etc. (Hample 1985/1992, 99). This reminds us of Robinson's conception, according to which one may understand argument $_{0}$ only if one understands the whole of cognition.

\footnotetext{
28 "In the everyday sense of the word, inference is a thought process during which from given knowledge we obtain new knowledge that has not been included explicitly [...] in the original knowledge. However, if inference is a mental activity, and logic deals with inferences, then the latter still studies certain aspects of thinking. We have no direct insight into thought processes, we can study only their linguistic manifestations. [...] The task of logic can be only the study of the relationship between the premises and the conclusion." (Ruzsa 2000, 10; our translation)
} 
Comparing our approach with these two views, it seems to be the case that our proposal can be treated either as an instance of the weak view according to which plausible inferences are objectifications of our own cultural knowledge about patterns of public inferences; or, alternatively, they are instances of the strong view, thus they can be interpreted as constituting a new element in Robinson's 'distributed system'. However, there may be a third and more convincing possibility as well:

\footnotetext{
"The weak view has a sterile conception of invention and seemingly little room for creativity. [...] Its portrait of the arguing person seems deterministic and only vaguely humane. [...] The strong view, on the other hand, enmeshes argument ${ }_{0}$ so thoroughly in psychology that arguing mentally ends up having no special character at all. An individual scholar need not maintain that one set of claims is superior to the other. One reasonable tactic is to try to shade one set into the other, in an attempt to resolve the tensions between them." (Hample 1985/1992, 100f; emphasis added)
}

In fact, this strongly resembles that kind of cyclic and prismatic inference procedures which we discussed in section $\mathbf{2 . 1}$ and applied in section 3 .

These observations can be generalized in the following way. On the one hand, the peculiarities of the linguistic level vastly underdetermine the cognitive level, because there will always be a lot of incompatible theories which claim to describe certain aspects of linguistic behaviour, while we know too little about the way cognition works. On the other hand, the cognitive level has to be considered fundamental. It is only natural therefore that a cyclic way of proceeding is required in the course of which we as researchers try to correlate the characteristics of the linguistic and cognitive level again and again, from different perspectives, looking for solutions which can resolve the inconsistencies between the hypotheses drawn from them. Thus, our answer to the question asked at the beginning of this section is clearly no.

Therefore, we are justified in interpreting the above quotation as supporting our hypothesis according to which theory formation in pragmatics proceeds along the same lines as plausible reasoning in general and implicatures in particular. That is, reasoning in the field of pragmatics can be characterized by the same properties we summarized with respect to the cognitive basis of pragmatic principles which underlie implicatures, namely,

(a) drawing inferences from a partial basis,

(b) using patterns of plausible inferences,

(c) the emergence and resolution of contradictions,

Acta Linguistica Hungarica 52, 2005 
(d) context-dependency,

(e) the continuous cyclic and prismatic revaluation of the information available.

There are two ways to evaluate this finding from a metascientific point of view. Firstly, the sub-problems of (P1) may turn out to be just manifestations of this kind of scientific reasoning - that is, (P1)(a)-(d) can be interpreted in terms of cyclic and prismatic reflection on the object of investigation rather than as mistakes such as the category error mentioned, the circularity of argumentation, interpretations with unexplicated criteria, or the fact that researchers objectify their own cultural knowledge as pragmatic principles. This kind of reinterpretation of the phenomena which Robinson labelled as methodological mistakes clearly legitimizes 'objective theories'. The point, then, is that theory formation in pragmatics works in a very different way (i.e., along the principles of plausible reasoning) from what is commonly assumed. Secondly, the application of cyclic and prismatic reasoning in the course of the construction of pragmatic theories may lead to the opposite result as well: namely, to realizing that these problematic cases are really errors. But in certain situations it is one of the primary functions of cyclic and prismatic reflection to rule out cases which are real mistakes and to distinguish them from the continuous revaluation of our scientific knowledge base.

\section{Conclusions}

As we saw in section 2.1, plausible reasoning is universal in that it works with respect to both everyday behaviour and scientific reasoning. Accordingly, it is the same principles which may be used to capture both object-scientific problems such as, for example, the nature of implicatures, and metascientific problems such as the nature of scientific reasoning. Nevertheless, the universality of plausible reasoning is not unlimited and must not mean uniformity. Rather, as the quotation in (viii) in section 2.1 emphasizes, the way plausible reasoning is manifested differs from domain to domain. Then, two crucial problems arise:

(a) How do the two cases of plausible reasoning we considered in this paper - i.e., as a constitutive component of the cognitive base of pragmatic principles and as a constitutive component of scientific reasoning — differ? 
(b) What is there in common between, on the one hand, drawing plausible inferences in everyday discourse such as the reasoning underlying implicatures, and, on the other, drawing plausible inferences in pragmatic theories whose task is to reveal these inferential mechanisms in everyday communication?

It goes without saying that these questions have to be left open for now and should be tackled by future considerations. Therefore, the line of argumentation put forward in this paper seems to pave the way for starting systematic investigations into the argumentational structure of linguistic theories - this is a field of which very little is known and that which is thought to be known, is probably easy to refute.

\section{Appendix: Patterns of plausible reasoning applied in section $3.2^{29}$}

\section{Shaded reduction}

It is possible that if $A$, then $B$.

It is possible that $B$.

After verifying $B, A$ is more credible.

It is possible that if $A$, then $B$.

It is possible that $B$.

After $B$ has become more credible, $A$ is more credible.

\section{Shaded modus tollens}

It is possible that if $A$, then $B$.

It is possible that $B$.

After $B$ has become less credible, $A$ is less credible.

It is possible that if $A$, then $B$.

Not $B$.

$A$ has become less credible.

\footnotetext{
${ }^{29}$ Other versions of these schemes are possible as well, varying in the degree of the
} plausibility of the different premises; cf. Polya (1954, 26); Rákosi (2005). 


\section{Shaded modus ponens}

It is possible that if $A$, then $B$.

It is possible that $A$.

After verifying $A, B$ is more credible.

It is possible that if $A$, then $B$.

It is possible that $A$.

After $A$ has become more credible, $B$ is more credible.

\section{Analogical inference}

$a_{1}$ has the properties $S$ and $T$

$a_{2}$ has the properties $S$ and $T$

$\cdots$

$a_{n}$ has the properties $S$ and $T$

$a_{n+1}$ has the property $S$

$a_{n+1}$ also has the property $T$

\section{References}

Benoit, William L. - Dale Hample - Pamela J. Benoit (eds) 1992. Readings in argumentation. Foris, Berlin \& New York.

Dolinina, Inga 2001. 'Theoretical' and 'empirical' reasoning modes from the neurological perspective. In: Argumentation 15:117-34.

Grice, H. Paul 1975. Logic and conversation. In: Peter Cole-Jerry L. Morgan (eds): Syntax and semantics, vol. 3: Speech acts, 41-58. Academic Press, New York.

Hample, Dale 1985/1992. A third perspective on argument. In: Benoit et al. (1992, 91116).

Kertész, András 2004a. Cognitive semantics and scientific knowledge. Case studies in the cognitive science of science. Benjamins, Amsterdam \& Philadelphia.

Kertész, András 2004b. Philosophie der Linguistik: Studien zur naturalisierten Wissenschaftstheorie. Narr, Tübingen.

Kertész, András - Csilla Rákosi 2005a. Inconsistency and plausible inferences in an analysis of German affricates. A case study in the philosophy of linguistics. In: Language Sciences (forthcoming).

Kertész, András - Csilla Rákosi 2005b. Whole-part and part-whole inferences in generative and cognitive linguistics. In: Acta Linguistica Hungarica (forthcoming).

Levinson, Stephen C. 2000. Presumptive meanings: The theory of generalized conversational implicature. MIT Press, Cambridge MA.

O'Keefe, Daniel J. 1977/1992. Two concepts of argument. In: Benoit et al. (1992, 79$90)$. 
Papafragou, Anna - Julien Musolino 2003. Scalar implicatures: Experiments at the semantics-pragmatics interface. In: Cognition 86:253-82.

Politzer, Guy - Gaëtan Bourmaud 2002. Deductive reasoning from uncertain conditionals. In: British Journal of Psychology $93: 345-81$.

Polya, George 1948. How to solve it. Princeton University Press, Princeton NJ.

Polya, George 1954. Patterns of plausible inference. Oxford University Press, Oxford.

Rákosi, Csilla 2005. Nyelvészeti elméletek argumentációs szerkezetének elemzése [The analysis of the argumentation structure of linguistic theories]. Doctoral dissertation, University of Debrecen.

Rescher, Nicholas 1976. Plausible reasoning. Van Gorcum, Assen \& Amsterdam.

Rescher, Nicholas 1987. How serious a fallacy is inconsistency? In: Argumentation 1: 303-16.

Rescher, Nicholas - Robert Brandom 1979. The logic of inconsistency. Blackwell, Cambridge MA \& Oxford.

Robinson, Edward A. 1997. The cognitive foundations of pragmatic principles: Implications for theories of linguistic and cognitive representation. In: Jan Nuyts-Eric Pederson (eds): Language and conceptualization, 253-71. Cambridge University Press, Cambridge.

Ruzsa, Imre 2000. Bevezetés a modern logikába [An introduction to modern logic]. Osiris Kiadó, Budapest.

Sperber, Dan - Deirdre Wilson 1986/1995. Relevance: communication and cognition. Blackwell, Cambridge MA \& Oxford. 1st/2nd edition.

Walton, Douglas 2001. Abductive, presumptive and plausible arguments. In: Informal Logic $21: 141-69$.

Woods, John - Andrew Irvine-Douglas Walton 2000. Argument: Critical thinking, logic and the fallacies. Pearson, Toronto.

Address of the authors: András Kertész-Csilla Rákosi

Research Group for Theoretical Linguistics

of the Hungarian Academy of Sciences

at the University of Debrecen

Pf. 47.

H-4010 Debrecen

Hungary

kert.esz@freemail.hu; rakosics@delfin.unideb.hu

Acta Linguistica Hungarica 52, 2005 